\title{
Hot Quark Matter with an Axial Chemical Potential
}

\author{
Raoul Gatto ${ }^{1}$ and Marco Ruggieri ${ }^{2}$ \\ ${ }^{1}$ Departement de Physique Theorique, Universite de Geneve, CH-1211 Geneve 4, Switzerland. \\ ${ }^{2}$ Department of Physics and Astronomy, University of Catania, Via S. Sofia 64, I-95125 Catania
}

\begin{abstract}
We analyze the phase diagram of hot quark matter in presence of an axial chemical potential, $\mu_{5}$. The latter is introduced to mimic the chirality transitions induced, in hot Quantum Chromodynamics, by the strong sphaleron configurations. In particular, we study the curvature of the critical line at small $\mu_{5}$, the effects of a finite quark mass and of a vector interaction. Moreover, we build the mixed phase at the first order phase transition line, and draw the phase diagram in the chiral density and temperature plane. We finally compute the full topological susceptibility in presence of a background of topological charge.
\end{abstract}

PACS numbers: 12.38.Aw, 12.38.Mh, 12.38.Lg

Keywords: Effective Models of QCD, Axial Chemical Potential, Phase Structure of QCD.

\section{INTRODUCTION}

Understanding the properties of strongly interacting matter, in extreme conditions of high temperature and/or large baryon density, is very important to get a deeper knowledge of our universe, at the macroscopic as well as at the microscopic levels. For example, very hot matter, with estimated temperature of the order of $10^{12}$ Kelvin, is produced in heavy ion collisions (HICs) experiments immediately after the collision, see for example [1] for an indirect measurement of such a temperature by the PHENIX collaboration at Brookhaven National Laboratory (BNL). As a consequence, it is crucial to make theoretical investigations on the phase structure of Quantum Chromodynamics (QCD), the theory of strong interactions, in conditions of high temperature, in order to give proper interpretation of experimental data, at the same time suggesting new phenomena to look for.

The theoretical knowledge on the thermodynamics of QCD at zero baryon density is fixed by Lattice simulations, which allow first principle numerical computations of the relevant thermodynamical quantities. In fact, Lattice simulations performed by independent groups show that in the range of temperature $(140,180) \mathrm{MeV}$, a crossover from the hadron phase to the deconfinement phase takes place, see [2, 3] and references therein. On the other hand, at finite baryon chemical potential it is not possible to use first principle Lattice simulations to investigate the structure of QCD (with three colors), because of the sign problem, see [4] for a review. Lattice simulations in the strong coupling limit seem promising to avoid the sign problem, see $[\underline{5}, \underline{6}$, even if the problem of the continuum limit within this approach has to be considered with care; in particular, the continuum limit is not reached in [5], but the $1 / g^{2}$ evolution in a range of $\beta=6 N_{c} / g^{2} \leq 4 \equiv \beta_{s}$ is consistent with Lattice Montecarlo simulations, for which the continuum limit is realized for $\beta \approx 6$, hence not too far from $\beta_{s}$.

The lack of feasible first principle calculations of the QCD thermodynamic properties at finite baryon chemical potential invokes the use of other theoretical strate- gies to investigate the structure of QCD in this regime. The main strategy is the use of some model. Among the several models, the Nambu-Jona-Lasinio (NJL) model [7] is very popular, see [8] for reviews. In the NJL model, the QCD gluon-mediated interactions are replaced by effective interactions among quarks, which are built in order to respect the global symmetries of QCD. Under some approximations, it is possible to derive the NJL model effective interaction kernel from first principles QCD, see 9, 10]. The common feature of the effective models is that they share (most of) the symmetries of the QCD Lagrangian. At the same time, they are able to describe spontaneous chiral symmetry breaking, as well as other kinds of breaking patterns expected at large chemical potential, in a self-consistent way. Moreover, it has been shown how it is possible to extend the chiral models in order to compute quantities which are sensible to confinemt properties of a given phase [11], see [12 24, 60, 61] for recent studies. One of the advantage of these models is that they allow for analytic or semi-analytic computations at zero as well as at finite chemical potential, at least at the one-loop level; thus they do not rely (in general) on simulations, and are not affected by the complex value of the quark determinant, allowing speculations about the structure of QCD in regimes where first principles calculations are not feasible. After standard bosonization procedure, the chiral and deconfinement transitions are described in terms of collective fields which take some expectation value, and whose quantum fluctuations describe physical particles ( $\sigma$ and $\pi$ mesons in the simplest version of the model).

It has been suggested that very strong magnetic fields are produced during the very first moments of a noncentral heavy ion collision [25 27]; this has motivated several studies about the effect of a strong magnetic background on the QCD phase structure, see [28 39] and references therein. Moreover, since the temperature of the fireball produced by the collision is very high, a copious production of topological gluon configurations (i.e., the QCD sphalerons) with finite winding number is expected [40], which induce locally chirality imbalance in the hot plasma as a natural consequence of the QCD 
Ward identity. The combined effect of the latter and of the magnetic field induces an electric current along the direction of the magnetic field. This effect, called the Chiral Magnetic Effect (CME) 25, 41], leads to the event-by-event separation of electric charges with respect to the reaction plane, which is a parity $(\mathcal{P})$ as well as a $\mathcal{C P}$-odd effect. Experimental data obtained by the STAR collaboration at BNL seem to point towards the direction of charge separation in collisions [42], even if the interpretation of such data in terms of the CME is still under debate [27, 43, 44].

Because of the expected production of chirality imbalance in the quark-gluon-plasma phase of QCD, and because of its potential relevance for the physics of heavy ion collisions, it is of interest to study how chirality modifies the structure of QCD itself. This is the main scope of this article. In particular, we continue the study of [4548] in which chirality was induced by an axial chemical potential, $\mu_{5}$, conjugated to chirality. Besides the applications to the CME, which are very interesting on their own because of the potential relevance for the phenomenology of heavy ion collisions, the theory at finite $\mu_{5}$ is interesting because it does not suffer from the sign problem; as a consequence, grandcanonical ensembles at finite $\mu_{5}$ can be simulated on the Lattice [49, 50]. These studies might be helpful to understand the structure of the QCD phase diagram at finite baryon chemical potential. For example, in [47] it has been suggested that the critical endpoint of the QCD phase diagram might be detected in Lattice simulations at finite $\mu_{5}$. The details about the theoretical framework will be given in Section II of the article. Here, we wish to stress the novelties embraced by our study.

Firstly, we investigate the effect of the vector interaction, as well as the finite current quark mass, on the location of the critical endpoint. Such aspects should be taken into account if a comparison with the Lattice data is desirable, but they have not been studied in [47]. Moreover, it is of interest to analyze analytically the effect of the axial chemical potential on the chiral condensate, and on the curvature of the critical line for restoration of chiral symmetry. The latter aspect is quite interesting, since it shows how a competition between the vacuum term and the thermal excitations compete and eventually lead to a lowering of the critical temperature. This reduction of the critical temperature was found numerically in the previous model studies [45 47] but it was not investigated in detail. Thirdly, we are interested to the phase diagram in the $n_{5}-T$ plane. This might be interesting because $n_{5}$ is connected to the topological charge density, because of the integrated Ward identity. With an abuse of nomenclature, we call the latter as canonical ensemble formulation, in analogy with the case of QCD at finite baryon density. Finally, we compute the full topological susceptibility at zero as well as at finite $\mu_{5}$, as a function of temperature. The latter part of our investigation has some overlap with the model study of Ref. [19], where topological susceptibility with a background of topolog- ical charge is computed. In the case of Ref. [19], the background topological charge is introduced by adding a $\theta$-term to the QCD action.

The plan of the paper is as follows. In Section II we introduce the axial chemical potential. In Section III we summarize the model we use in our calculations. In Section IV we perform a perturbative analysis at small $\mu_{5}$ at zero as well as at finite temperature, computing the dependence of the chiral condensate and of the critical temperature on the axial chemical potential. In Section $\mathrm{V}$ we relate the phase diagrams in the canonical and grand-canonical ensembles, and build explicitly the mixed phase. In Section VI we study the effect of the bare quark mass, and of a vector interaction, on the location of the critical endpoint of the phase diagram in the $\mu_{5}-T$ plane. Finally, we present our conclusions in Section VII.

\section{THE AXIAL CHEMICAL POTENTIAL}

In this Section we define the axial chemical potential, $\mu_{5}$. It has been already discussed in several references, see [41, 45 47, 51] and references therein. Therefore we limit ourselves to the basic definitions and to fix our notation. First of all we introduce the chirality, $N_{5}=N_{R}-N_{L}$, as the imbalance between right- and left-handed quarks. In QCD, change of chirality at zero as well as at finite temperature can be related directly to the topology of nonperturbative gluon configurations with a finite winding number, $Q_{W}$, via the integrated Ward identity,

$$
N_{5}=n_{5} V=2 N_{f} Q_{W},
$$

where $N_{f}$ is the number of flavors considered, and we have assumed that before the interaction with the gluon configuration, chirality was zero. In Equation (11) we have introduced the chiral density $n_{5}$. The above equation is a consequence of the strong chiral anomaly; $Q_{W}$ is related to the topological charge of the given gluon configuration,

$$
Q_{W}=\frac{g^{2}}{32 \pi^{2}} \int d^{4} x F_{a}^{\mu \nu} \tilde{F}_{\mu \nu}^{a} .
$$

At high temperature, a copious production of gluon configurations with nonvanishing winding number is expected, see for example [40] and references therein. These are the strong (that is, QCD) sphalerons. Therefore, because of the existence of QCD sphalerons, chirality can be produced in the high temperature phase of QCD.

The simplest way to treat quark matter with net chirality in effective models, is to introduce an axial chemical potential, $\mu_{5}$, conjugated to chiral density [41, 45 47]. At the lagrangian level, this amounts to add the chiral density operator,

$$
\mu_{5} \bar{\psi} \gamma^{0} \gamma^{5} \psi
$$

to the lagrangian density. This procedure is similar to the one usually adopted to study systems at finite baryon 
density: in the latter case, we introduce a baryon chemical potential, $\mu$, that induces a net baryon density, $n$. For example, in the very high temperature phase of two flavor QCD, the following relation holds [41]:

$$
n_{5}=\frac{\mu_{5}^{3}}{3 \pi^{2}}+\frac{\mu_{5} T^{2}}{3},
$$

which shows how a chiral chemical potential induces a net chirality in the system. In the general case, one needs to compute the relation between $n_{5}$ and $\mu_{5}$ numerically, see [45, 46] and Section V of this article.

Before going ahead, it is necessary to make few remarks. Firstly, we are aware that the axial chemical potential cannot be considered as a true chemical potential. As a matter of fact, $\mu_{5}$ is conjugated to $n_{5}$, or to the topological charge because of the Ward identity. The latter is a not conserved quantity in QCD because of the quantum anomaly. In the common picture of the QCD vacuum with $\mu_{5}=0$, the vanishing average value of the topological charge is understood as the formation of several local domains, each one characterized by a finite topological charge; the probability to create a domain with charge $Q_{W}$ is the same as the probability to create a domain with charge $-Q_{W}$. As a consequence, in a volume much larger than the typical domain size, the average value of the topological charge sums up to zero. However, flucuations of the topological charge can change the value of the charge of any local domain. The formal role of the axial chemical potential is to reproduce the local domains of the QCD vacuum with a net topological charge as equilibrium states. Because of the fluctuations of the topological charge, this description is meaningful as long as the time scale is shorter than the inverse of the topological changing transition rate [49]. This interesting theoretical question has been analyzed also in [52], where it is claimed that a proper combination of the chiral density and of a Chern-Simon term is a conserved quantity, and it is precisely this quantity that should be coupled to the axial chemical potential. We will devote a more detailed study of this problem to a next article.

Another remark is that the theory with $\mu_{5} \neq 0$ is a sign-free theory. It is well known that QCD with three colors suffers the sign problem: namely, the fermion determinant of QCD with three colors is complex at finite quark chemical potential, making the usual Montecarlo sampling of configurations in the Lattice simulations not possible when the quark chemical potential is larger than the temperature (see [4] for a review). On the other hand, the theory at finite $\mu_{5}$ does not suffer the sign problem. As a matter of fact, $\gamma_{5} D\left(\mu_{5}\right) \gamma_{5}=D^{\dagger}\left(\mu_{5}\right)$, where $D$ corresponds to the Dirac operator with $\mu_{5} \neq 0$. As a consequence, the fermion determinant is real and positive at $\mu_{5} \neq 0$, and grand canonical ensembles with finite $\mu_{5}$ can be simulated on the Lattice [41]. Indeed, some Lattice simulation at $\mu_{5} \neq 0$ has been already performed to study the chiral magnetic effect on the Lattice, see [49, 50]. In these references, a preliminary study of the phase structure in the $\mu_{5}-T$ plane is also addressed.

\section{THE MODEL}

In this Section, we describe the model that we use in calculations, namely the Nambu-Jona-Lasinio model improved with the Polyakov loop (PNJL in the following) [11]. In the PNJL model, quark propagation in the medium is described by the following lagrangian density:

$$
\mathcal{L}=\bar{q}\left(i \gamma^{\mu} D_{\mu}-m\right) q+G\left[(\bar{q} q)^{2}+\left(i \bar{q} \gamma_{5} \tau q\right)^{2}\right] ;
$$

here $q$ is the quark Dirac spinor in the fundamental representation of the flavor $S U(2)$ and the color group; $\boldsymbol{\tau}$ correspond to the Pauli matrices in flavor space. A sum over color and flavor is understood. The covariant derivative embeds the QCD coupling with the background gluon field which is related to the Polyakov loop, see below.

The PNJL model has access to the expectation value of the Polyakov loop, $L$, which is sensible to confinement or deconfinement properties of a given phase. In order to compute $\langle L\rangle$ we introduce a static, homogeneous and Euclidean background temporal gluon field, $A_{0}=i A_{4}=$ $i \lambda_{a} A_{4}^{a}$, coupled minimally to the quarks via the QCD covariant derivative. Then $W=\operatorname{Tr}_{c} \exp \left(i \beta \lambda_{a} A_{4}^{a}\right)$ and $L=W / 3$, where $\beta=1 / T$. In the Polyakov gauge, which is convenient for this study, $A_{0}=i \lambda_{3} \phi+i \lambda_{8} \phi^{8}$; moreover, for simplicity we take $L=L^{\dagger}$ from the beginning as in [12], which implies $A_{4}^{8}=0$.

In our computation we follow the idea implemented in 21], which brings to a Polyakov-loop-dependent coupling constant:

$$
G=g\left[1-\alpha_{1} L L^{\dagger}-\alpha_{2}\left(L^{3}+\left(L^{\dagger}\right)^{3}\right)\right],
$$

The ansatz in the above equation was inspired by [9] in which it was shown explicitly that the NJL vertex can be derived in the infrared limit of QCD, it has a non-local structure, and it acquires a non-trivial dependence on the phase of the Polyakov loop. This idea has been analyzed recently in [53], where the effect of the confinement order parameter on the four-fermion interactions and their renormalization-group fixed-point structure has been investigated. The numerical values of $\alpha_{1}$ and $\alpha_{2}$ have been fixed in 21 by a best fit of the available Lattice data at zero and imaginary chemical potential of Ref. [54, 55]. In particular, the fitted data are the critical temperature at zero chemical potential, and the dependence of the Roberge-Weiss endpoint on the bare quark mass. The best fit procedure leads to $\alpha_{1}=\alpha_{2} \equiv \alpha=0.2$, which are the values we adopt in this article.

In the one-loop approximation, the effective potential of this model is given by [47]

$$
\begin{aligned}
V= & \mathcal{U}\left(L, L^{\dagger}, T\right)+\sigma^{2} G-N_{c} N_{f} \sum_{s= \pm 1} \int \frac{d^{3} \boldsymbol{p}}{(2 \pi)^{3}} \omega_{s} \\
& -\frac{N_{f}}{\beta} \sum_{s= \pm 1} \int \frac{d^{3} \boldsymbol{p}}{(2 \pi)^{3}} \log \left(F_{+} F_{-}\right),
\end{aligned}
$$


where $\sigma=\bar{q} q$ is a scalar collective field representing the mean field and the quantum fluctuations of the operator $\bar{q} q$, and $G$ is defined in Eq. (6) ; moreover,

$$
\omega_{s}=\sqrt{\left(|\boldsymbol{p}| s-\mu_{5}\right)^{2}+m_{q}^{2}},
$$

corresponds to the pole of the quark propagator, and $m_{q}=m-2 \sigma G$ is the constituent quark mass; the index $s$ denotes the helicity projection. In Eq. (17) we have introduced the functions

$$
\begin{aligned}
& F_{-}=1+3 L e^{-\beta\left(\omega_{s}-\mu\right)}+3 L^{\dagger} e^{-2 \beta\left(\omega_{s}-\mu\right)}+e^{-3 \beta\left(\omega_{s}-\mu\right)}, \\
& F_{+}=1+3 L^{\dagger} e^{-\beta\left(\omega_{s}+\mu\right)}+3 L e^{-2 \beta\left(\omega_{s}+\mu\right)}+e^{-3 \beta\left(\omega_{s}+\mu\right)},
\end{aligned}
$$

which are responsible for the statistical confinement property of the model at low temperature [11].

In right hand side of the first line of Equation (17) the momentum integral corresponds to the vacuum quark fluctuations contribution to the thermodynamic potential. We treat the divergence in this term phenomenologically, introducing a momentum cutoff, $\Lambda$, in the vacuum term; the numerical value of $\Lambda$ will be then fixed by requiring that the quark condensate, the pion mass and the pion decay constant computed in the model are in agreement with the phenomenological values. Before going ahead, it is useful to remind that within the quarkmeson model a renormalization procedure is feasible [17], the model itself being renormalizable. The renormalizability of the model might be useful to remove the cutoff effects that, instead, appear in the NJL model (see the discussion in the next Sections). On the other hand, the renormalization program of the energy density at finite $\mu_{5}$ is not trivial, since a nonvanishing $\mu_{5}$ induces further divergences in the theory, as it can be argued by an inspection of the $\mu_{5}$-dependence of the vacuum energy, see for example Eq. (14). Hence, proper renormalization conditions should be adopted.

The potential term $\mathcal{U}$ in Eq. (7) is built by hand in order to reproduce the pure gluonic lattice data with $N_{c}=3[12]$. We adopt the following logarithmic form,

$$
\begin{aligned}
& \mathcal{U}[L, \bar{L}, T]=T^{4}\left\{-\frac{a(T)}{2} \bar{L} L\right. \\
& \left.+b(T) \ln \left[1-6 \bar{L} L+4\left(\bar{L}^{3}+L^{3}\right)-3(\bar{L} L)^{2}\right]\right\}
\end{aligned}
$$

with three model parameters (one of four is constrained by the Stefan-Boltzmann limit),

$$
\begin{aligned}
& a(T)=a_{0}+a_{1}\left(\frac{T_{0}}{T}\right)+a_{2}\left(\frac{T_{0}}{T}\right)^{2}, \\
& b(T)=b_{3}\left(\frac{T_{0}}{T}\right)^{3} .
\end{aligned}
$$

The standard choice of the parameters reads $a_{0}=3.51$, $a_{1}=-2.47, a_{2}=15.2$ and $b_{3}=-1.75$. The parameter
$T_{0}$ in Eq. (11) sets the deconfinement scale in the pure gauge theory. In absence of dynamical fermions one has $T_{0}=270 \mathrm{MeV}$. However, dynamical fermions induce a dependence of this parameter on the number of active flavors [16]. For the case of two light flavors to which we are interested here, we take $T_{0}=190 \mathrm{MeV}$ as in [21]. Also for the remaining parameters we follow [21] and take $\Lambda=631.5 \mathrm{MeV}, m=5.5 \mathrm{MeV}$ and $g=5.498 \times 10^{-6}$ $\mathrm{MeV}^{-2}$.

We notice that the PNJL model considered here, which is dubbed Extended-PNJL in [21], has been tuned in order to reproduce quantitatively the Lattice QCD thermodynamics at zero and imaginary quark chemical potential. Hence, it represents a faithful description of QCD, in terms of collective degrees of freedom related to chiral symmetry breaking and deconfinement.

\section{PERTURBATIVE ANALYSIS}

In the case of small $\mu_{5}$, we can make some analytical and semianalytical estimate of the effect of the axial chemical potential on quark condensation, and on the critical line. In this Section we restrict our analysis to the pure NJL model case, corresponding to take $L=L^{\dagger}=1$ in Eq. (7), and $\alpha_{1}=\alpha_{2}=0$ in Eq. (6). This simplifies the numerical analysis. On the other hand, the results obtained here will not be modified qualitatively by the Polyakov loop, since the effect of the latter is just a suppression of colored states below $T_{c}$. Moreover, we work in the chiral limit; this simplification allows to define rigorously the chiral phase transition, and compute unambiguously the critical temperature.

\section{A. Zero temperature: chiral condensate}

To begin with, we consider the zero temperature case, and we compute the shift of the chiral condensate induced by $\mu_{5}$, showing that the chiral chemical potential acts as a catalyzer of chiral symmetry breaking. In fact, the $\mu_{5}$-dependent zero temperature correction to the effective potential is given by

$$
V_{1}^{0}=-\frac{N_{c} N_{f}}{2 \pi^{2}} \mu_{5}^{2} m_{q}^{2} F\left(\frac{m_{q}}{\Lambda}\right)
$$

where we have assumed $\mu_{5} \ll m_{q}$; in Eq. (14) we have introduced the function

$$
F(x)=\log \frac{1+\sqrt{1+x^{2}}}{x}-\frac{1}{\sqrt{1+x^{2}}} .
$$

It is easy to prove that $F(x)$ is always positive, thus making $V_{1}^{0}<0$. Hence, the energy density of a broken phase and $\mu_{5} \neq 0$ is smaller than that of a phase with unbroken symmetry and the same value of the chiral condensate.

We can use Eq. (14) to analyze the perturbative solution of the gap equation at zero temperature. As a matter 
of fact, for small values of $\mu_{5}$ we can look for a solution of the gap equation in the form $m_{q}=\bar{m}_{q}+\delta m_{q}$, where $\bar{m}_{q}$ satisfies the gap equation at $\mu_{5}=0$, and $\delta m_{q}$ corresponds to the $\mu_{5}$-dependent contribution. An elementary computation shows that

$$
\delta m_{q}=-\left.\frac{1}{M_{\sigma}^{2}}\left(\frac{\partial V_{1}^{0}}{\partial m_{q}}\right)\right|_{m_{q}=\bar{m}_{q}},
$$

where we have defined $M_{\sigma}^{2} \equiv\left(\partial^{2} V_{0} / \partial m_{q}^{2}\right)_{m_{q}=\bar{m}_{q}}$ and $V_{0} \equiv V\left(\mu_{5}=0\right)$. By definition, $M_{\sigma}^{2}>0$, since $\bar{m}_{q}$ corresponds to a minimum of the effective potential at $\mu_{5}=0$. Moreover, for $m_{q} \ll \Lambda$ we find $\left(\partial V_{1}^{0} / \partial m_{q}\right) \propto$ $m_{q} \log \left(m_{q} / \Lambda\right)$ which is negative (we have verified that the sign does not change as long as $m_{q} \leq \Lambda$ ). We conclude that $\delta m_{q}>0$, showing that $\mu_{5}$ favors the spontaneous breaking of chiral symmetry at zero temperature.

\section{B. Finite temperature: chiral condensate}

We can extend the analysis of the previous subsection, to the case of finite temperature. For the perturbative solution of the gap equation, the derivative of the effective potential with respect to the quark mass is needed, see Eq. (16). At finite temperature, the $\mu_{5}$-dependent contribution of the effective potential is $V_{1}=V_{1}^{0}+V_{1}^{T}$ with $V_{1}^{0}$ given by Eq. (14). Thus, Eq. (16) is replaced by

$$
\delta m_{q}=-\left.\frac{1}{M_{\sigma}^{2}}\left(\frac{\partial V_{1}^{0}}{\partial m_{q}}+\frac{\partial V_{1}^{T}}{\partial m_{q}}\right)\right|_{m_{q}=\bar{m}_{q}} .
$$

The expression of $V_{1}^{T}$ is not informative, thus it is not necessary to report it here. Its derivative with respect to the quark mass is more interesting,

$$
\frac{\partial V_{1}^{T}}{\partial m_{q}}=N_{c} N_{f} \mu_{5}^{2} G\left(m_{q}, T\right) .
$$

In the above equation, $G\left(m_{q}, T\right)$ corresponds to a function defined in terms of a convergent numerical integral. In Fig. 1, we plot $G$ as a function of temperature, for two different values of the quark mass. We find that $G>0$. As a consequence, because of Eq. (17), the $\mu_{5}$-dependent contribution of the thermal fluctuations tends to reduce the value of the constituent quark mass (i.e., of the chiral condensate).

\section{Critical line}

We compute in this subsection the critical temperature, $T_{c}$, as a function of $\mu_{5}$, and show at the same time that the chiral phase transition is of the second order. To this end we perform an expansion of the effective potential near $T_{c}$. In fact, the order parameter around $T_{c}$ is small enough that a series expansion in powers of $\sigma / T$ is justified. Since we restrict ourselves to the case of

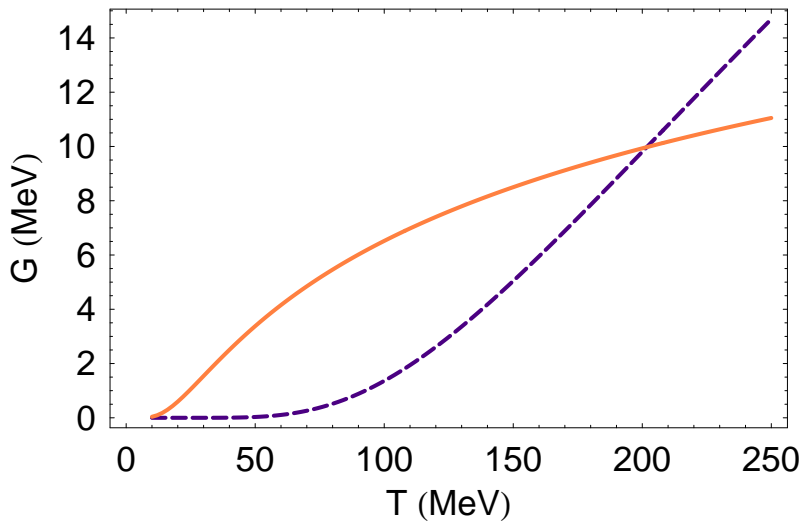

FIG. 1. Function $G$ of Eq. (18) versus temperature, for two values of the constituent quark mass, $m_{q}$. Orange solid line corresponds to $m_{q}=50 \mathrm{MeV}$; Indigo dashed line corresponds to the case $m_{q}=350 \mathrm{MeV}$.

the pure NJL model, there is no dependence from the Polyakov loop in the NJL coupling constant, $G$.

One can write

$$
V=V_{0}+\frac{\alpha_{2}}{2} G^{2} \sigma^{2}+\frac{\alpha_{4}}{4} G^{4} \sigma^{4}+\ldots,
$$

where the dots denote higher order terms; $V_{0}$ is the potential at $\sigma=0$ : it is independent on the condensate, thus it is just a number which does not affect the physics of the problem. We notice that because of our definition of the $\sigma$ field, which has the dimension of a cubic mass, we have extracted the proper powers of the NJL coupling constant from the definitions of the coefficients, in order to give the common mass dimension to the coefficients themselves. At the second order transition point $T=T_{c}$ one has $\alpha_{2}=0$ and $\alpha_{4}>0$. Therefore, to determine the critical temperature as a function of $\mu_{5}$ it is enough to determine the zeros of $\alpha_{2}$ in the $\mu_{5}-T$ plane. Next, we compute $\alpha_{4}$ to check that the transition points correspond to a second order phase transition.

Computing the second derivative of the effective potential in Equation (7) we find, after some algebra,

$$
\alpha_{2}=\alpha_{2,0}+\alpha_{2,2} \mu_{5}^{2},
$$

where

$$
\begin{aligned}
\alpha_{2,0} & =\frac{2}{G}-\frac{2 N_{c} N_{f}}{\pi^{2}} \Lambda^{2}+\frac{2 N_{c} N_{f}}{3} T^{2}, \\
\alpha_{2,2} & =\frac{4 N_{c} N_{f}}{\pi^{2}}\left(\log \frac{T}{\Lambda}+c\right),
\end{aligned}
$$

and the numerical factor $c=1.36$ arises from a convergent numerical integral.

At small $\mu_{5}$ the equation $\alpha_{2}=0$, which determines the critical temperature, can be solved perturbatively: from the condition $\alpha_{2,0}=0$ we find

$$
T_{c}^{0}=\left(\frac{3 \Lambda^{2}}{\pi^{2}}-\frac{3}{N_{c} N_{f} G}\right)^{1 / 2} ;
$$


using the parameters of the model we find $T_{c}^{0}=174 \mathrm{MeV}$. Then, writing $T_{c}=T_{c}^{0}+\delta T$ and solving $\alpha_{2,0}+\alpha_{2,2} \mu_{5}^{2}=0$ for $\delta T$ we find

$$
T_{c}=T_{c}^{0}\left(1-\frac{3 \mu_{5}^{2}}{4 N_{c} N_{f}\left(T_{c}^{0}\right)^{2}} \alpha_{2,2}\left(T_{c}^{0}\right)\right) .
$$

Using the parameters given above we find $\alpha_{2,2}\left(T_{c}^{0}\right)=$ 0.17 , which implies that the critical temperature is a decreasing function of $\mu_{5}$. Hence, a finite $\mu_{5}$ favors the disordered phase at finite temperature.

The last step is to check the sign of $\alpha_{4}$, to be confident that the transition is of the second order. Since we make a perturbative analysis at $\mu_{5} \ll T$, we may assume $\mu_{5}$ infinitesimal of the same order of $\sigma$, hence terms of the order of $\mathcal{O}\left(\mu_{5}^{2} \sigma^{4}\right)$ can be regarded as $\mathcal{O}\left(\sigma^{6}\right)$ and can be neglected. At the leading order, it is therefore enough to compute the coefficient at $\mu_{5}=0$,

$$
\alpha_{4}=\frac{48 N_{c} N_{f}}{\pi^{2}}\left(\log \frac{\Lambda}{T}-b\right),
$$

where $b=0.90$ arises from a convergent integral computed numerically. Using the values of $\Lambda$ and $T_{c}^{0}$ we find that $\alpha_{4}=11.35$ at $T=T_{c}^{0}$; being it positive, the phase transition is of the second order.

Before going ahead, it is useful to summarize the results of this Section. Eq. (17) represents the correction to the solution of the gap equation due to $\mu_{5} \neq 0$. The first and second addenda in the right hand side of the equation correspond to the zero temperature and finite temperature contributions, respectively. At zero temperature, only the first contribution survives, and it is negative, leading to a positive shift of the constituent quark mass. On the other hand, the contribution of thermal fluctuations is positive. Thus at a given temperature, there is a competition between the effect of $\mu_{5}$ on the vacumm and thermal contributions to the gap equation. In passing, we notice that if vacuum fluctuations were neglected in the thermodynamic potential, then the thermal contribution in Eq. (17) would lower the constituent quark mass at finite temperature as $\mu_{5}$ is switched on. Finally, the results in Equations (22), (24) and (25) show that at zero and small $\mu_{5}$, the chiral phase transition is of the second order, and the critical temperature is a decreasing function of the chiral chemical potential. This scenario for the critical line is in agreement with the results of $45-$ 47], where a numerical procedure of minimization of the one-loop potential has been adopted.

\section{GRANDCANONICAL AND CANONICAL PHASE DIAGRAMS}

In this Section, we describe the expected phase structure of QCD at finite temperature and axial chemical potential, as predicted by the PNJL model. The grandcanonical phase diagram, already discussed in [47], corresponds to the map of the different phases in the $\mu_{5}-T$ plane. On the other hand, for the case of the canonical ensemble phase diagram, $\mu_{5}$ is replaced by the chiral density $n_{5}$, the latter being defined as

$$
n_{5}=-\frac{\partial \Omega}{\partial \mu_{5}},
$$

where the derivative has to be computed at the global minimum of the grand potential.

The computation of $n_{5}$ in the model needs some care. In particular, at $m_{q} \neq 0$ the divergence of the vacuum energy at finite $\mu_{5}$ is transmitted to $n_{5}$. As a matter of fact, $n_{5}$ can be formally split into a vacuum and a thermal parts. The thermal part is convergent, and its derivative is finite as well, therefore it does not lead to any difficulty. On the other hand, the $T=0$ contribution is divergent, and its derivative with respect to $\mu_{5}$ is divergent as well in the case $m_{q} \neq 0$. This can be realized easily if we take for a moment the limit $\Lambda \gg \mu_{5}, m_{q}$, in which we find

$$
n_{5}=\frac{N_{c} N_{f}}{2 \pi^{2}}\left[\frac{2}{3} \mu_{5}^{3}+2 m_{q}^{2} \mu_{5}\left(\log \frac{2 \Lambda}{m_{q}}-1\right)\right] .
$$

In the case $m_{q}=0$ we obtain the result of [41], which is not affected by the ultarviolet divergence. Because of the latter, we cannot take the limit $\Lambda \rightarrow \infty$ in the calculation of the phase space integrals which are involved in the expressions of $n_{5}$. Therefore in the numerical computation of $n_{5}$, we cutoff the vacuum contribution at $p=\Lambda$ unless otherwise stated, for internal consistency.

Before the discussion of the results, it is useful to comment briefly on the expected effect of the finite cutoff on $n_{5}$. If $\mu_{5} \gg m_{0}$, which is appropriate in the quarkgluon plasma phase, we can neglect the mass term in the vacuum contribution of the fermion determinant to $n_{5}$, which we call $\mathcal{C}_{0}$ :

$$
\mathcal{C}_{0}=\frac{N_{c} N_{f}}{3 \pi^{2}}\left[\mu_{5}^{3} \theta\left(\Lambda-\mu_{5}\right)+\Lambda^{3} \theta\left(\mu_{5}-\Lambda\right)\right] .
$$

We notice that the term proportional to $\Lambda^{3}$ in the above equation does not appear in Eq. (27) since in the latter we have taken the limit $\Lambda \gg \mu_{5}$. As soon as $\mu_{5}>\Lambda$, the vacuum contribution to the chiral density saturates because of the existence of a cutoff in the theory, as shown by Eq. (28). In the numerical calculations, we expect that the saturation effect will be softened by the thermal fluctuation contributions. As a consequence we expect to measure some mild saturation effect as $\mu_{5} \approx \Lambda$. This saturation is observed on the Lattice as well, see [49, 50]. In last analysis, this is not a serious trouble, since we expect the model to be consistent only when masses are smaller than the cutoff, in this case for regime $\mu_{5}<\Lambda$.

In Fig. 2 we plot the normalized chiral density, $\rho_{W} \equiv$ $n_{5} / 2 N_{f}$, as a function of $\mu_{5}$ for several values of the temperature. In the Figure, $T_{c}=173.9 \mathrm{MeV}$ corresponds to the critical temperature for deconfinement and chiral symmetry restoration at $\mu_{5}=0$. At large values of $\mu_{5}$ we measure the expected saturation of $\rho_{W}$. This saturation is not physical but a mere artifact of our regularization 


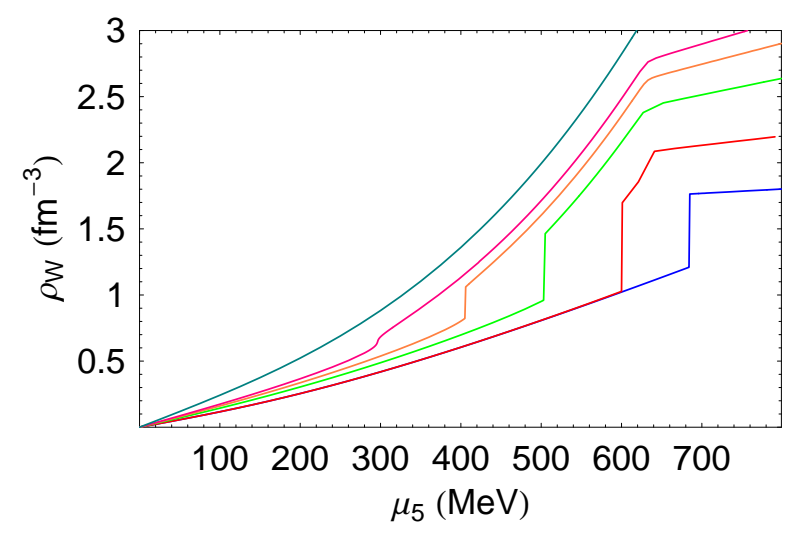

FIG. 2. Normalized chiral density, $\rho_{W} \equiv n_{5} / 2 N_{f}$, as a function of the chiral chemical potential, at several values of the temperature. From right to left, temperature is equal to $0.4 T_{c}, 0.6 T_{c}, 0.8 T_{c}, 0.9 T_{c}, T_{c 5}, 1.1 T_{c}$ respectively.

scheme. Hence we will consider trustable only the results in the range $\mu_{5}<\Lambda$; when the results outside the above specified range are shown, it is done only for completeness.

Next we turn to the phase structure. The grandcanonical phase diagram has been computed in [47]. It is useful, however, to briefly summarize here the results of [47], in order to facilitate the comparison with the canonical phase diagram. In the upper panel of Fig 3 we plot the phase diagram in the $\mu_{5}-T$ plane. The indigo dashed line corresponds to the chiral and deconfinement crossover; the solid line denotes the first order phase transition. The indigo dot is the critical endpoint. The scale $T_{c}=173.9$ $\mathrm{MeV}$ corresponds to the critical temperature at $\mu_{5}=0$.

The critical line in the phase diagram in Fig. 3 is identified with the peak of the derivative $d L / d T$. Within few $\mathrm{MeV}$, we have found that the latter coincides with the location of the peak of $|d \sigma / d T|$, for the values of $\mu_{5}$ analyzed in this work. Thus, within the model ad hand, the deconfinement and the chiral symmetry restoration take place simultaneously. In last analysis, this is related to the explicit dependence of the NJL coupling on the Polyakov loop, and to the mechanism that leads to deconfinement in the model. As a matter of fact, at finite temperature the one- and two-quark contributions to the grand potential act as efficient $Z_{3}$-breaking terms, which in turn favor the $L \neq 0$ state. As a consequence, the effective value of the NJL coupling which is responsible for the spontaneous chiral symmetry breaking is reduced with respect to the zero temperature case, as soon as $L \neq 0$, see Eq. (6). Thus, it is expected that deconfinement in the extended PNJL model implies chiral symmetry restoration, as it is confirmed by the numerical results. The inverse assertion, namely that chiral symmetry restoration implies deconfinement, is not true and indeed it is not necessarily realized in the model, unless an explicit backreaction on the Polyakov loop effective potential is introduced, see for example [14, 16, 24].
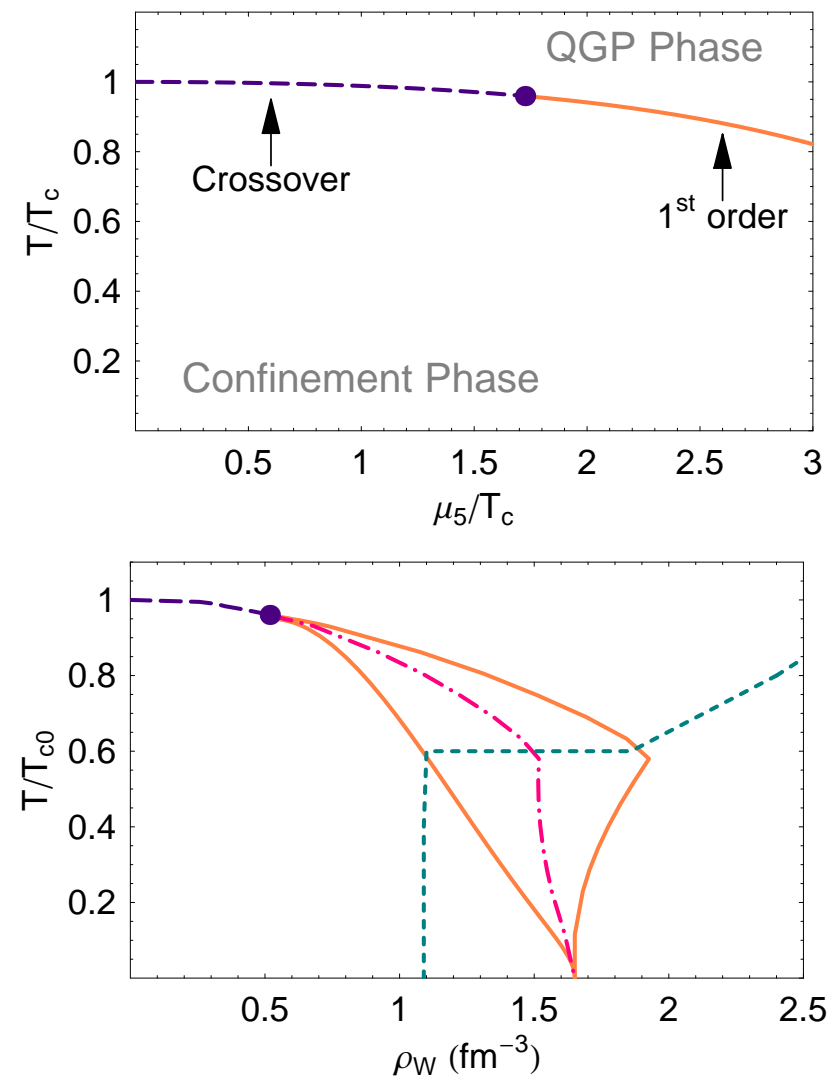

FIG. 3. (color online). Upper panel: phase diagram in the grandcanonical ensemble. The indigo dashed line corresponds to the chiral and deconfinement crossover; the solid line denotes the first order phase transition. The indigo dot is the critical endpoint. The scale $T_{c}=173.9 \mathrm{MeV}$ corresponds to the critical temperature at $\mu_{5}=0$. Lower panel: phase diagram in the temperature-chiral density plane. Solid lines correspond to the boundaries of the mixed phase. On the left line the volume fraction, $c$, of hadron phase is $c=1$; on the right line we find $c=0$. Dashed line denotes the chiral and deconfinement crossovers. Dot-dashed line corresponds to the values of $T\left(\rho_{W}\right)$ at which $c=1 / 2$. In the portion of the phase diagram below the dotted line we find $\mu_{5}>\Lambda$. Finally, the indigo dot denotes the critical endpoint.

The grandcanonical phase diagram can be translated to a canonical one, by replacing $\mu_{5}$ with $\rho_{W}$. This program is easily accomplished once we use Eq. (26) to compute $\rho_{W}$, once the physical values of $\langle\bar{q} q\rangle$ and $L$ are known. As already explained in the Introduction, the use of the term canonical has to be taken as an abuse of nomenclature. Indeed $n_{5}$ is not a conserved quantity, because of the fluctuations of the topological charge in QCD and the Ward identity. Thus, discussing about $n_{5}$ is meaningful only if the time of observation of the system is smaller than the typical time needed to observe a fluctuation of the topological charge, the latter being related to the inverse of the sphaleron transition rate.

In the lower panel of Fig. 3 we plot the phase diagram in the $\rho_{W}-T$ plane. In the figure, the indigo dashed 
line denotes the chiral and deconfinement crossovers (the two coincide within numerical uncertainties within this model calculation). The indigo dot denotes the critical endpoint. Its coordinates in the phase diagram are

$$
\rho_{5}^{c}=0.518 \mathrm{fm}^{-3}, \quad T_{5}^{c}=167 \mathrm{MeV} .
$$

The orange solid lines correspond to the boundaries of the mixed phase, which develops in correspondence of the first order phase transition line of the grancanonical phase diagram. The composition of the mixed phase at the temperature $T$ can be evaluated easily as follows. Given the total charge density $\rho_{W}$, then the volume fractions of the ordered, $c$, and disordered, $d$, phases have to satisfy $c \rho_{1}+d \rho_{2}=\rho_{W}$ with $c+d=1$; here $\rho_{1,2}$ denote the values of the topological charge density on the two boundaries of the mixed phase region. It follows then

$$
c=\frac{\rho_{2}-\rho_{W}}{\rho_{2}-\rho_{1}} ;
$$

on the left line the volume fraction $c=1$, corresponding to a homogeneous pure ordered phase; on the right line $c=0$, corresponding to a pure disordered phase. For reference, in Fig. 3 we plot a pink dot-dashed line which corresponds to the values of $T\left(\rho_{W}\right)$ at which $c=1 / 2$. Finally, in the portion of the phase diagram below the green dotted line we find $\mu_{5}>\Lambda$, in which cutoff artifacts are relevant. At small temperatures the green line is almost vertical, because the topological charge density turns out to be very insensitive of temperature, see Fig.2.

\section{INFLUENCE OF THE QUARK MASS AND OF THE VECTOR INTERACTION}

In this Section, we wish to investigate the quantitative effect of a nonphysical bare quark mass, as well as of the presence of the vector interaction, on the critical line in the $\mu_{5}-T$ plane. In particular, we wish to compute the evolution of the critical endpoint coordinates as a function of the bare quark mass, and of the coupling strength in the vector channel.

\section{A. The effect of the bare quark mass}

In this Section, we study the effects of the bare quark mass on the location of the critical endpoint $\mathrm{CP}_{5}$. Estimation of the effect of the bare quark mass is very important, because Lattice simulations at $\mu_{5} \neq 0$ nowadays are performed with a numerical value of the quark mass which is larger than the physical value. In Lattice simulations, the non physical value of the quark mass is reflected into a non physical value of the pion mass. In [49, 50], the value of the pion mass in the vacuum is $m_{\pi} \approx 400$ $\mathrm{MeV}$.

In Fig. 4 we plot the critical endpoint coordinates as a function of the bare quark mass. In the figure, the solid

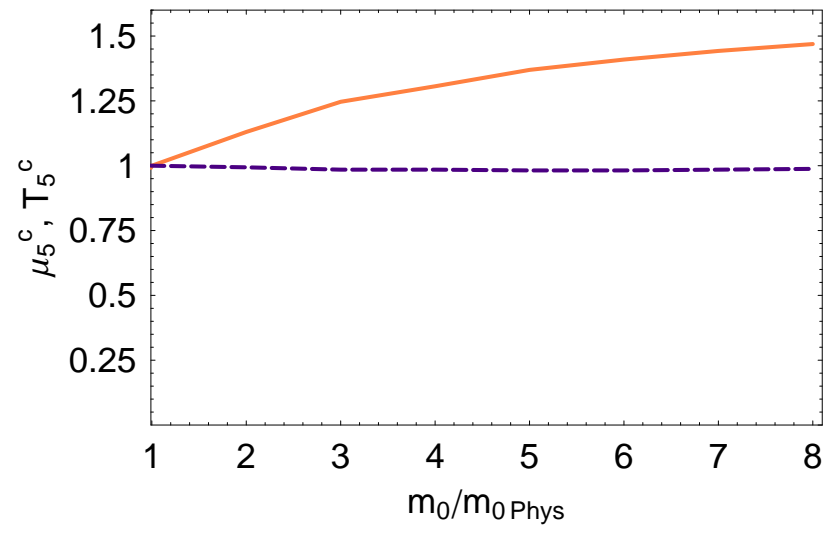

FIG. 4. (Color online). Evolution of the critical endpoint coordinates as a function of the bare quark mass. Solid orange line corresponds to the critical value of the axial chemical potential; dashed indigo line corresponds to the critical endpoint temperature. Axial chemical potential and temperature are measured in units of the endpoint coordinates at $m_{0}=m_{0, \text { Phys }}$.

orange line corresponds to the critical value of the axial chemical potential; the dashed indigo line corresponds to the critical endpoint temperature. Axial chemical potential and temperature are measured in units of the endpoint coordinates at $m_{0}=m_{0 \text {,Phys }}$ with $m_{0 \text {,Phys }}=5.5$ $\mathrm{MeV}$, which corresponds to $m_{\pi} \approx 139 \mathrm{MeV}$. For comparison, at the largest value of quark mass considered here we find $m_{\pi} \approx 400 \mathrm{MeV}$.

Our results show that the critical endpoint temperature, $T_{5}^{c}$, is not so much affected by the quark mass. As we will show in the next Section, this inertia of $T_{5}^{c}$ is also present when we switch on a vector interaction. On the other hand, the critical endpoint axial chemical potential, $\mu_{5}^{c}$, is strongly affected by the quark mass. In more detail, the larger value of $m_{0}$, the larger $\mu_{5}^{c}$. This is quite easy to understand naively, since the bare quark mass turns the chiral transition to a crossover. At $\mu_{5}=0$, the chiral crossover is smoothed as $m_{0}$ is increased. Therefore, it is natural to expect that a larger value of $\mu_{5}$ is necessary to experience a first order phase transition, if $m_{0}>m_{0 \text {,Phys }}$. This might partly explain why the critical endpoint $\mathrm{CP}_{5}$ is not yet detected in Lattice simulations [49, 50].

\section{B. The role of a vector interaction}

In this Section we briefly comment on the role of a vector interaction on the phase structure of the model. To this end, we add to the lagrangian density the term

$$
\mathcal{L}_{V}=-G_{V}\left[\left(\bar{\psi} \gamma^{\mu} \psi\right)^{2}+\left(\bar{\psi} \gamma^{\mu} \gamma_{5} \psi\right)^{2}\right]
$$

we do not include the interaction in the triplet channel, since at the one-loop level it gives rise to terms which couple the isospin density, $\delta n=n_{d}-n_{u}$, to the isospin 
density operator; these are not relevant as long as we do not introduce the isospin chemical potential.

At the mean field level one has

$$
\begin{aligned}
\mathcal{L}_{V}= & G_{V} n^{2}-2 G_{V} n \bar{\psi} \gamma^{0} \psi \\
& +G_{V} n_{5}^{2}-2 G_{V} n_{5} \bar{\psi} \gamma^{0} \gamma^{5} \psi ;
\end{aligned}
$$

as usual, we have defined $n=\left\langle\psi^{\dagger} \psi\right\rangle$ and $n_{5}=\left\langle\psi^{\dagger} \gamma^{5} \psi\right\rangle$. Thus at the mean field level, the chemical potentials for quark number density and for chiral density are shifted because of the vector interaction,

$$
\begin{gathered}
\mu \rightarrow \mu-2 G_{V} n, \\
\mu_{5} \rightarrow \mu_{5}-2 G_{V} n_{5} .
\end{gathered}
$$

The previous equations are useful to grasp the effect of $G_{V} \neq 0$ on the phase structure. As a matter of fact, if $G_{V}>0$ then quarks propagate in a thermal bath at an affective chemical potential which is smaller than the true chemical potential, because of Eq. (33). As a consequence, the transition at finite $\mu$ is smoothed, and the critical endpoint moves to higher values of the chemical potential. On the same footing, because of Eq. (34), the effective chiral chemical potential is smaller than $\mu_{5}$. Therefore we expect that the critical endpoint coordinate moves to higher values of $\mu_{5}$ compared to the case $G_{V}=0$.

This reasonings are confirmed by our concrete numerical computations. In the case of $G_{V} \neq 0$, the value of $n_{5}$ has to be computed self-consistently by means of the number equation (26), which has to be solved, at any given value of $\mu_{5}$ and $T$, together with the gap equations,

$$
\frac{\partial \Omega}{\partial \sigma}=\frac{\partial \Omega}{\partial L}=0 .
$$

In Fig. 5 we plot the expectation value of the Polyakov loop in the pseudocritical range as a function of temperature, for several values of the strength of the coupling in the vector channel, at $\mu_{5}=300 \mathrm{MeV}$. At $G_{V}=0$, this value of $\mu_{5}$ is slightly above $\mu_{5}^{c}$. In the figure, solid line corresponds to $G_{V}=0$; dashed line corresponds to $G_{V}=0.25 G$; dot-dashed line corresponds to $G_{V}=0.5 G$; dotted line corresponds to $G_{V}=0.75 G$; finally, short dashed line corresponds to $G_{V}=G$. As expected, the role of the vector interaction is to smooth the phase transition in comparison with the latter at $G_{V}=0$. Indeed, the first order phase transition at $\mu_{5}=300 \mathrm{MeV}$ at $G_{V}=0$ is turned by the vector interaction into a crossover at the same value of $\mu_{5}$.

In Fig. 6 we plot the critical endpoint coordinates, $\mu_{5}^{c}$ and $T^{c}$, as a function of the ratio $G_{V} / G$. Blue dashed line corresponds to the critical temperature; red solid line corresponds to the critical chiral chemical potential. We find that the temperature of the critical endpoint is poorly affected by the value of $G_{V}$ in the range that we have examined; on the other hand, the critical value of the chiral chemical potential is quite sensitive to the vector coupling.

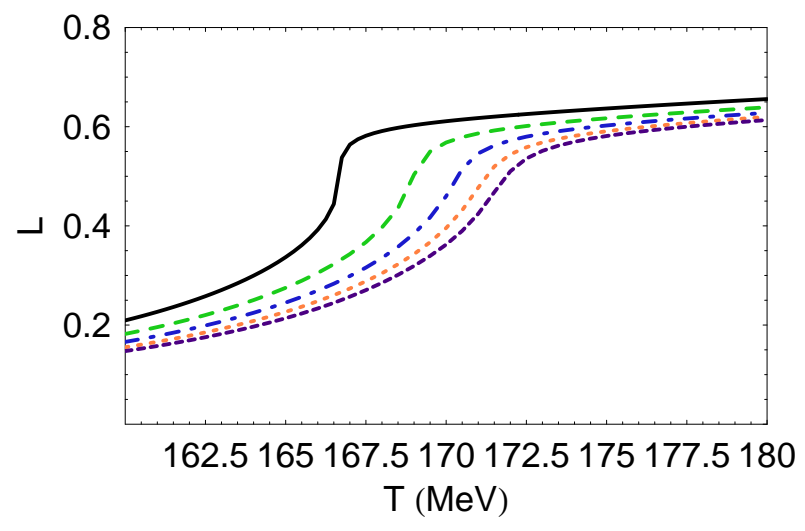

FIG. 5. (Color online). Expectation value of the Polyakov loop in the pseudocritical range as a function of temperature, for several values of the strength of the coupling in the vector channel, at $\mu_{5}=300 \mathrm{MeV}$. Black solid line corresponds to $G_{V}=0$. Green dashed line corresponds to $G_{V}=0.25 G$. Blue dot-dashed line corresponds to $G_{V}=0.5 G$. Orange dotted line corresponds to $G_{V}=0.75 G$. Finally, indigo short dashed line corresponds to $G_{V}=G$.

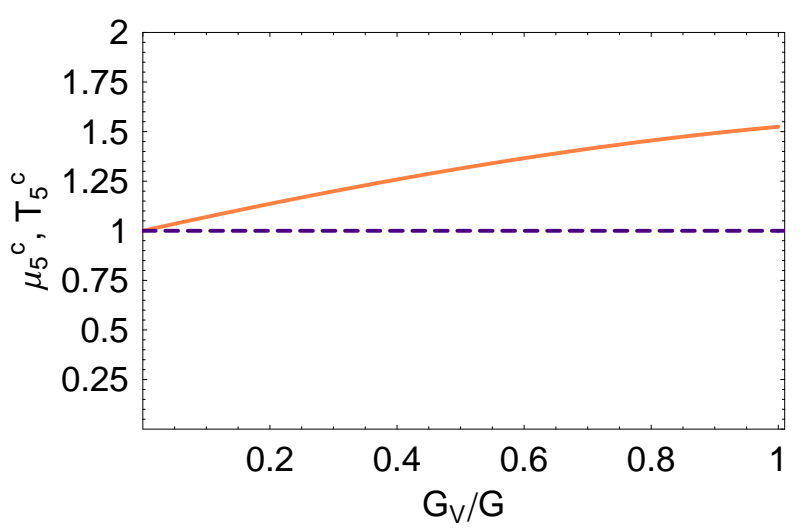

FIG. 6. (Color online). Critical endpoint coordinates as a function of the ratio $G_{V} / G$. Indigo dashed line corresponds to the critical temperature; orange solid line corresponds to the critical chiral chemical potential. Axial chemical potential and temperature are measured in units of the endpoint coordinates at $m_{0}=m_{0, \text { Phys }}$.

It is useful to comment about the similarities between the phase structure at finite $\mu_{5}$, which turns out from our computation, and the one well established (within effective models) at finite baryon chemical potential, $\mu$. In this article, we have considered only one type of condensate, namely the chiral condensate, which characterizes the symmetry breaking pattern at finite $T$ and $\mu_{5}$ in the fermion sector of our model. Besides a smaller curvature of the critical line in the case of the chiral chemical potential, compared with that at finite $\mu$, we do not find qualitative difference in the phase structure. Also, the effect of the vector interaction, as well as of a finite current 
quark mass, is very similar, qualitatively, in the cases of finite $\mu$ and finite $\mu_{5}$. Some difference between the two phase diagrams might arise from different types of condensates. For example, an important point that we have not considered in our article, for the sake of simplicity, is the introduction of a diquark condensate, which might appear at finite mu5. For the case of QCD at finite $\mu$, such a condensate is expected to be developed at very large $\mu$ 62 64]. However, this possibility deserves further study and needs to be checked numerically by dynamical computation of the condensates. We leave this point to a future project.

\section{TOPOLOGICAL SUSCEPTIBILITY}

In this Section we compute the topological susceptibility at finite temperature, in presence of a background chiral density. Topological susceptibility in QCD is defined as the correlator of the topological charge at zero momentum; it can be computed from the QCD partition function via the relation

$$
\chi=\left.\frac{\partial^{2} \Omega}{\partial \theta^{2}}\right|_{\theta=0},
$$

where the $\theta$-angle is introduced by adding the following term to the lagrangian density:

$$
\theta \frac{g^{2}}{64 \pi^{2}} \varepsilon^{\mu \nu \rho \sigma} F_{\mu \nu}^{a} F_{\rho \sigma}^{a}
$$

It is well known that one can get rid of the term in Eq. (37) in the QCD action by means of a chiral rotation. However, after the chiral transformation, the $\theta$ dependence of the QCD partition function appears explicitely in the quark part of the QCD action. A model study having some overlap with our study can be found in [19], where the full topological susceptibility in presence of a background of topological charge is computed. In the case of [19], the topological charge is introduced by adding the $\theta$-term to the lagrangian density, which in turns acts as a source for the topological charge. The relevance of a finite $\theta$ in [19] lies on the possibility that for temperatures higher than $\Lambda_{Q C D}$, sphaleron transitions induce a spacetime dependent $\theta$-angle [51].

A low energy relation connects the vacuum chiral condensate to the topological susceptibilty; in the theory of $N_{f}$ light flavors the relation reads [56, 57]

$$
\chi=|\langle\bar{q} q\rangle|\left(\sum_{f} \frac{1}{m_{f}}\right)^{-1}+O\left(\frac{m_{f}}{\Lambda_{Q C D}}\right)
$$

where $\langle\bar{q} q\rangle$ is the common value of the quark condensates for the light quarks. Equation (38) shows that the topological susceptibility is proportional to the product of the light flavor masses; thus, in the equation it is manifest the fact that in a theory in which at least one massless

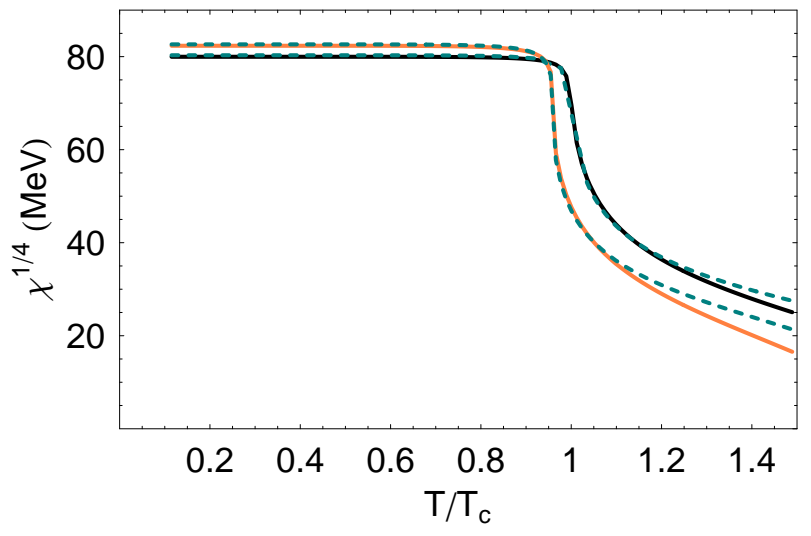

FIG. 7. Topological susceptibility as a function of temperature, for several values of $\mu_{5}$. Black data correspond to $\mu_{5}=0$; orange data correspond to $\mu_{5}=300 \mathrm{MeV}$. The green dotted lines correspond to the right hand side of Eq. (38).

flavor exists, one has $\chi=0$. This was proved also by Ward identities in 58.

As stated before, a chiral rotation transmits the $\theta$ dependence of the QCD action to the quark sector. After the rotation, the quarks acquire a complex mass term. Besides, the condensates that are not invariant under the axial rotation are mixed among themselves. This scenario can be implemented within the effective models to compute $\theta$-dependent quantities, see for example [59]. Beside this, the $\theta$-dependent action can be used to compute $\chi$ by virtue of Eq. (36). A detailed discussion about the implementation of the $\theta$-dependent lagrangian within the chiral model with the Polyakov loop can be found in [19, 20]. In this article it is enough to mention that, in order to introduce the $\theta$ angle in the model and compute the topological susceptibility, it is enough to change $M=m_{0}-2 \sigma G$ into the grand potential with

$$
\sqrt{\left[m_{0} \cos \left(\frac{\theta}{2}\right)-2 \sigma G\right]^{2}+m_{0}^{2} \sin ^{2}\left(\frac{\theta}{2}\right)}
$$

It is worth to mention here that generally speaking, at finite $\theta$ other condensates might develop (and indeed they do develop, see [19, 20]). The presence of these condensates makes the substitution (39) not sufficient for the complete treatment of the $\theta$ angle within the chiral models. However, such new condensates vanish in the theory at $\theta=0$, to which we are interested; neglecting them from the beginning does not change the derivative of $\Omega$ at $\theta=0$. Therefore, the replacement in Eq. (39) is sufficient for the purpose of computing the topological susceptibility within this model.

In Fig. 7 we plot the topological susceptibility as a function of temperature, for several values of $\mu_{5}$. At zero temperature and chiral chemical potential we find

$$
\chi=(79.97 \mathrm{MeV})^{4},
$$


which is in agreement with the large $N_{c}$ prediction in Eq. (38), which gives $\chi=(80.3 \mathrm{MeV})^{4}$ with two light flavors at $T=\mu_{5}=0$.

The relation (38) is actually satisfied within the chiral model also at $T=0$ and $\mu_{5} \neq 0$. Indeed, a straightforward computation shows that

$$
\chi=-\frac{m_{0}}{2} \frac{N_{c} N_{f}}{2 \pi^{2}} \sigma \mathcal{I}
$$

where

$$
\mathcal{I}=G \int_{0}^{\Lambda}\left[\frac{p^{2} d p}{\sqrt{\left(p+\mu_{5}\right)^{2}+\left(m_{0}-2 \sigma G\right)}}+\mu_{5} \rightarrow-\mu_{5}\right]
$$

moreover, from the conditions $\partial \Omega / \partial \sigma=0$ and $\sigma=2\langle\bar{u} u\rangle$ we have

$$
\langle\bar{u} u\rangle=-\frac{N_{c} N_{f}}{2 \pi^{2}} \sigma \mathcal{I}+O\left(\frac{m_{0}}{m_{q}}\right)
$$

Here $m_{q}$ corresponds to the constituent quark mass. A comparison between Eqs. (41) and (43) leads to

$$
\chi=-\frac{m_{0}}{2}\langle\bar{u} u\rangle+O\left(\frac{m_{0}}{m_{q}}\right)
$$

which is in agreement with Eq. (38) when the latter is computed for two degenerate flavors. On the other hand, at finite temperature we measure some discrepancy between the theoretical prediction given by Eq. (38), corresponding to the green dotted line in Fig. 7, and the numerical results obtained within the PNJL model (solid lines in the same figure).

\section{CONCLUSIONS}

In this article, we have reported our new results about the structure of hot quark matter in a background of a chiral density, the latter induced by a finite axial chemical potential $\mu_{5}$. Firstly, we have analyzed analytically the effect of the axial chemical potential on the chiral condensate, and on the critical temperature for restoration of chiral symmetry. We restricted ourselves to the case of the NJL model, and to the chiral limit. However, even within these simplifications, we are able to understand the shape of the critical line at finite $\mu_{5}$.
Secondly, we have discussed the phase diagram in the canonical ensemble formulation, in which $\mu_{5}$ is replaced by the chiral density, $n_{5}$. This might be interesting because $n_{5}$ in QCD is connected locally to the topological charge density, because of the integrated Ward identity. As a consequence, it might be of interest to predict the numerical value of the topological charge density along the critical line, as well as at the critical endpoint.

Thirdly, we have computed the effect of the vector interaction, as well as of the finite current quark mass, on the location of the critical endpoint. Such aspects should be taken into account if a comparison with the Lattice data is desirable, and extend the study started in [47].

As a final investigation, we have computed the full topological susceptibility (i.e., which takes into account both the pure gauge and the dynamical fermion contribution) at zero as well as at finite $\mu_{5}$, as a function of temperature. We find that the Di Vecchia-LeutwylerSmilga-Veneziano (DLSV) relation [56, 57] is satisfied at finite $\mu_{5}$ in the confinement phase of the model. At large temperature, above the critical temperature, we measure a deviation from the DLSV relation, both at zero and at finite $\mu_{5}$; this can be understood within the model, since terms of the order of $m_{0} / m_{q}$ which are negligible in the confinement phase, become important in the quarkgluon-plasma phase.

It is interesting to ask wether the work presented here can be improved. In our opinion, several directions are possible for future research. As a first step, it would be important to investigate from a theoretical point of view, how to couple correctly $\mu_{5}$ to a conserved quantity in QCD. Along this line, the work in Ref. 52] seems quite enlightening. Secondly, it is of interest to include the possibility of other condensates, among them a diquark condensate, in analogy to the situation of QCD at very large density. We plan to report on these, as well as related, topics in the next future.

\section{ACKNOWLEDGMENTS}

We acknowledge stimulating discussions with $\mathrm{P}$. de Forcrand, M. D'Elia, G. Endrodi, M. Frasca, K. Fukushima, F. Negro, O. Philipsen, about the topics discussed in this article. Moreover, we thank T. Z. Nakano, A. Ohnishi, Y. Sakai, T. Sasaki and H. Ueda for their careful reading of the manuscript and their useful comments. M. R. also acknowledges the Japanese Society for the Promotion of Science for support during his staying at Kyoto University, where par of this work was completed.
[1] A. Adare et al. [ PHENIX Collaboration ], Phys. Rev. C81, 034911 (2010).

[2] Y. Aoki, G. Endrodi, Z. Fodor, S. D. Katz, K. K. Szabo, Nature 443, 675-678 (2006); Y. Aoki, Z. Fodor,
S. D. Katz, K. K. Szabo, Phys. Lett. B643, 46-54 (2006); Y. Aoki, S. Borsanyi, S. Durr, Z. Fodor, S. D. Katz, S. Krieg, K. K. Szabo, JHEP 0906, 088 (2009); S. Borsanyi et al. [ Wuppertal-Budapest Collaboration ], JHEP 
1009, 073 (2010); S. Borsanyi, G. Endrodi, Z. Fodor, A. Jakovac, S. D. Katz, S. Krieg, C. Ratti, K. K. Szabo, JHEP 1011, 077 (2010); G. Endrodi, Z. Fodor, S. D. Katz, K. K. Szabo, JHEP 1104, 001 (2011).

[3] M. Cheng, N. H. Christ, S. Datta, J. van der Heide, C. Jung, F. Karsch, O. Kaczmarek, E. Laermann et al., Phys. Rev. D74, 054507 (2006); C. E. Detar et al. [ HotQCD Collaboration ], PoS LAT2007, 179 (2007); F. Karsch, PoS LAT2007, 015 (2007); F. Karsch [ RBC and HotQCD Collaborations ], J. Phys. G G35, 104096 (2008); A. Bazavov, T. Bhattacharya, M. Cheng, N. H. Christ, C. DeTar, S. Ejiri, S. Gottlieb, R. Gupta et al., Phys. Rev. D80, 014504 (2009); M. Cheng, S. Ejiri, P. Hegde, F. Karsch, O. Kaczmarek, E. Laermann, R. D. Mawhinney, C. Miao et al., Phys. Rev. D81, 054504 (2010);

[4] P. de Forcrand, PoS LAT2009, 010 (2009).

[5] T. Z. Nakano, K. Miura, A. Ohnishi, Phys. Rev. D83, 016014 (2011).

[6] P. de Forcrand, M. Fromm, Phys. Rev. Lett. 104, 112005 (2010).

[7] Y. Nambu and G. Jona-Lasinio, Phys. Rev. 122, 345 (1961); Y. Nambu and G. Jona-Lasinio, Phys. Rev. 124, 246 (1961).

[8] U. Vogl and W. Weise, Prog. Part. Nucl. Phys. 27, 195 (1991); S. P. Klevansky, Rev. Mod. Phys. 64, 649 (1992); T. Hatsuda and T. Kunihiro, Phys. Rept. 247, 221 (1994); M. Buballa, Phys. Rept. 407, 205 (2005).

[9] K. I. Kondo, Phys. Rev. D 82, 065024 (2010).

[10] M. Frasca, arXiv:1105.5274 [hep-ph]].

[11] K. Fukushima, Phys. Lett. B591, 277-284 (2004); P. N. Meisinger and M. C. Ogilvie, Phys. Lett. B 379, 163 (1996).

[12] S. Roessner, C. Ratti and W. Weise, Phys. Rev. D 75, 034007 (2007); C. Sasaki, B. Friman and K. Redlich, Phys. Rev. D 75, 074013 (2007); S. K. Ghosh, T. K. Mukherjee, M. G. Mustafa and R. Ray, Phys. Rev. D 77, 094024 (2008); A. Bhattacharyya, P. Deb, S. K. Ghosh and R. Ray, Phys. Rev. D 82, 014021 (2010).

[13] K. Fukushima, Phys. Rev. D 77, 114028 (2008) [Erratum-ibid. D 78, 039902 (2008)]; M. Ciminale, R. Gatto, N. D. Ippolito, G. Nardulli and M. Ruggieri, Phys. Rev. D 77, 054023 (2008); W. j. Fu, Z. Zhang and Y. x. Liu, Phys. Rev. D 77, 014006 (2008); T. Hell, S. Rossner, M. Cristoforetti and W. Weise, Phys. Rev. D 81, 074034 (2010).

[14] H. Abuki, R. Anglani, R. Gatto, G. Nardulli and M. Ruggieri, Phys. Rev. D 78, 034034 (2008).

[15] K. Kashiwa, H. Kouno, M. Matsuzaki and M. Yahiro, Phys. Lett. B 662, 26 (2008); Y. Sakai, T. Sasaki, H. Kouno and M. Yahiro, Phys. Rev. D 82, 076003 (2010).

[16] T. K. Herbst, J. M. Pawlowski and B. J. Schaefer, arXiv:1008.0081 [hep-ph].

[17] V. Skokov, B. Friman, E. Nakano, K. Redlich and B. J. Schaefer, Phys. Rev. D 82, 034029 (2010).

[18] T. Kahara and K. Tuominen, Phys. Rev. D 78, 034015 (2008); Phys. Rev. D 80, 114022 (2009); arXiv:1006.3931 [hep-ph].

[19] Y. Sakai, H. Kouno, T. Sasaki, M. Yahiro, arXiv:1105.0413 [hep-ph]].

[20] H. Kouno, Y. Sakai, T. Sasaki, K. Kashiwa, M. Yahiro, Phys. Rev. D83, 076009 (2011).
[21] Y. Sakai, T. Sasaki, H. Kouno and M. Yahiro, Phys. Rev. D 82, 076003 (2010).

[22] V. Skokov, B. Friman, K. Redlich, arXiv:1008.4570 [hepph]]; V. Skokov, B. Friman, E. Nakano, K. Redlich, B. J. Schaefer, Phys. Rev. D82, 034029 (2010).

[23] J. O. Andersen, R. Khan, L. T. Kyllingstad, arXiv:1102.2779 [hep-ph]].

[24] Y. Sakai, T. Sasaki, H. Kouno, M. Yahiro, arXiv:1104.2394 [hep-ph]].

[25] D. E. Kharzeev, L. D. McLerran, H. J. Warringa, Nucl. Phys. A803, 227-253 (2008).

[26] V. Skokov, A. Y. .Illarionov, V. Toneev, Int. J. Mod. Phys. A24, 5925-5932 (2009).

[27] V. Voronyuk, V. D. Toneev, W. Cassing, E. L. Bratkovskaya, V. P. Konchakovski, S. A. Voloshin, Phys. Rev. C83, 054911 (2011).

[28] M. D'Elia, S. Mukherjee, F. Sanfilippo, Phys. Rev. D82, 051501 (2010).

[29] M. D’Elia, F. Negro, Phys. Rev. D83, 114028 (2011).

[30] P. V. Buividovich, M. N. Chernodub, E. V. Luschevskaya, M. I. Polikarpov, Phys. Lett. B682, 484-489 (2010).

[31] A. J. Mizher, M. N. Chernodub, E. S. Fraga, Phys. Rev. D82, 105016 (2010).

[32] R. Gatto, M. Ruggieri, Phys. Rev. D82, 054027 (2010); Phys. Rev. D83, 034016 (2011).

[33] N. O. Agasian, S. M. Fedorov, Phys. Lett. B663, 445-449 (2008).

[34] E. S. Fraga, A. J. Mizher, Phys. Rev. D78, 025016 (2008).

[35] M. N. Chernodub, Phys. Rev. D82, 085011 (2010); Phys. Rev. Lett. 106, 142003 (2011).

[36] V. V. Braguta, P. V. Buividovich, M. N. Chernodub, M. I. Polikarpov, arXiv:1104.3767 [hep-lat]].

[37] N. O. Agasian, arXiv:1109.5849 [hep-ph]].

[38] N. Callebaut, D. Dudal, H. Verschelde, arXiv:1105.2217 [hep-th]].

[39] K. Fukushima, Phys. Rev. D83, 111501 (2011).

[40] G. D. Moore, M. Tassler, JHEP 1102, 105 (2011).

[41] K. Fukushima, D. E. Kharzeev, H. J. Warringa, Phys. Rev. D78, 074033 (2008).

[42] B. I. Abelev et al. [ STAR Collaboration ], Phys. Rev. Lett. 103, 251601 (2009); Phys. Rev. C81, 054908 (2010).

[43] K. Fukushima, D. E. Kharzeev, H. J. Warringa, Nucl. Phys. A836, 311-336 (2010).

[44] B. Muller, A. Schafer, Phys. Rev. C82, 057902 (2010).

[45] K. Fukushima, M. Ruggieri and R. Gatto, Phys. Rev. D 81, 114031 (2010).

[46] M. N. Chernodub, A. S. Nedelin, Phys. Rev. D83, 105008 (2011).

[47] M. Ruggieri, Phys. Rev. D84, 014011 (2011).

[48] C. A. B. Bayona, K. Peeters, M. Zamaklar, JHEP 1106, 092 (2011).

[49] A. Yamamoto, Phys. Rev. Lett. 107, 031601 (2011).

[50] A. Yamamoto, arXiv:1106.5479 [hep-lat]; arXiv:1108.0937 [hep-lat].

[51] L. D. McLerran, E. Mottola, M. E. Shaposhnikov, Phys. Rev. D43, 2027-2035 (1991); H. B. Nielsen, M. Ninomiya, Phys. Lett. B130, 389 (1983); A. N. Sisakian, O. Y. .Shevchenko, S. B. Solganik, hep-th/9806047; M. Joyce, T. Prokopec, N. Turok, Phys. Rev. D53, 29582980 (1996).

[52] V. A. Rubakov, arXiv:1005.1888 [hep-ph]]. 
[53] J. Braun, A. Janot, arXiv:1102.4841 [hep-ph]].

[54] M. D'Elia and F. Sanfilippo, Phys. Rev. D 80, 111501 (2009).

[55] C. Bonati, G. Cossu, M. D'Elia and F. Sanfilippo, arXiv:1011.4515 [hep-lat].

[56] G. Veneziano, Nucl. Phys. B159, 213-224 (1979); P. Di Vecchia, G. Veneziano, Nucl. Phys. B171, 253 (1980).

[57] H. Leutwyler, A. V. Smilga, Phys. Rev. D46, 5607-5632 (1992).

[58] R. J. Crewther, Phys. Lett. B70, 349 (1977).

[59] T. Fujihara, T. Inagaki, D. Kimura, Prog. Theor. Phys. 117, 139-160 (2007); D. Boer, J. K. Boomsma, Phys.
Rev. D78, 054027 (2008); J. K. Boomsma, D. Boer, Phys. Rev. D80, 034019 (2009).

[60] E. Megias, E. Ruiz Arriola and L. L. Salcedo, Phys. Rev. D 74, 114014 (2006)

[61] E. Megias, E. Ruiz Arriola and L. L. Salcedo, Phys. Rev. D 74, 065005 (2006)

[62] M. G. Alford, K. Rajagopal and F. Wilczek, Phys. Lett. B 422, 247 (1998)

[63] R. Rapp, T. Schafer, E. V. Shuryak and M. Velkovsky, Phys. Rev. Lett. 81, 53 (1998)

[64] M. G. Alford, K. Rajagopal and F. Wilczek, Nucl. Phys. B 537, 443 (1999) 